\title{
Genetic mapping and candidate gene identification of BoGL5, a gene essential for cuticular wax biosynthesis in broccoli
}

Fengqing $\mathrm{Han}^{\dagger}$, Jingjing Huang ${ }^{\dagger}$, Qi Xie, Yumei Liu, Zhiyuan Fang, Limei Yang, Mu Zhuang, Yangyong Zhang, Honghao LV, Yong Wang, Jialei Ji and Zhansheng Li

\begin{abstract}
Background: The aerial organs of most terrestrial plants are covered by cuticular waxes, which impart plants a glaucous appearance and play important roles in protecting against various biotic and abiotic stresses. Despite many glossy green (wax-defective) mutants being well characterized in model plants, little is known about the genetic basis of glossy green mutant in broccoli.

Results: B156 is a spontaneous broccoli mutant showing a glossy green phenotype. Detection by scanning electron microscopy (SEM) and chromatography-mass spectrometry (GC-MS) revealed that B156 is a cuticular wax-defective mutant, lacking waxes mostly longer than C28. Inheritance analysis revealed that this trait was controlled by a single recessive gene, BoGL5. Whole-genome InDel markers were developed, and a segregating $F_{2}$ population was constructed to map BoGL5. Ultimately, BoGL5 was mapped to a $94.1 \mathrm{~kb}$ interval on C01. The BOCER2 gene, which is homologous to the Arabidopsis CER2 gene, was identified as a candidate of BoGL5 from the target interval. Sequence analyses revealed that Bocer2 in B156 harbored a G-to-T SNP mutation at the 485th nucleotide of the CDS, resulting in a W-to-L transition at the 162nd amino acid, a conserved site adjacent to an HXXXD motif of the deduced protein sequence. Expression analysis revealed that BoCER2 was significantly down-regulated in the leaves, stems, and siliques of B156 mutant than that of B3. Last, ectopic expression of BOCER2 in A. thaliana could, whereas Bocer2 could not, rescue the phenotype of cer2 mutant.
\end{abstract}

Conclusions: Overall, this study mapped the locus determining glossy phenotype of B156 and proved BoCER2 is functional gene involved in cuticular wax biosynthesis which would promotes the utilization of BoCER2 to enhance plant resistance to biotic and abiotic stresses, and breeding of B. oleracea cultivars with glossy traits.

Keywords: Cuticular wax, Broccoli, Fine mapping, Candidate gene, Functional analysis

\section{Background}

The surfaces of terrestrial plants are covered by cuticles that serve as protective barriers between plants and the environment [1]. The cuticle consists of cutin, polysaccharides and cuticular waxes [2] and plays important

\footnotetext{
* Correspondence: lizhansheng@caas.cn

${ }^{\dagger}$ Fengqing Han and Jingjing Huang contributed equally to this work. Institute of Vegetables and Flowers, Chinese Academy of Agricultural Sciences, Key Laboratory of Biology and Genetic Improvement of Horticultural Crops, Ministry of Agriculture, \#12 Zhong Guan Cun Nandajie Street, Beijing 100081, China
}

roles in protecting plants against water loss, ultraviolet radiation, deposits of pollutants, and both bacterial and fungal pathogens [1]. In addition, the cuticle also affects plant-insect interactions [3].

Plant cuticular waxes comprise very long chain fatty acids (VLCFAs) and their derivatives, including C24 to C32 alkanes, aldehydes, alcohols, ketones and esters [4]. In the endoplasmic reticulum (ER) of plant epidermal cells, multienzymatic fatty acid elongase (FAE) complexes [5] catalyze elongation by adding two carbons per

(c) The Author(s). 2021 Open Access This article is licensed under a Creative Commons Attribution 4.0 International License, which permits use, sharing, adaptation, distribution and reproduction in any medium or format, as long as you give appropriate credit to the original author(s) and the source, provide a link to the Creative Commons licence, and indicate if changes were made. The images or other third party material in this article are included in the article's Creative Commons licence, unless indicated otherwise in a credit line to the material. If material is not included in the article's Creative Commons licence and your intended use is not permitted by statutory regulation or exceeds the permitted use, you will need to obtain permission directly from the copyright holder. To view a copy of this licence, visit http://creativecommons.org/licenses/by/4.0/ The Creative Commons Public Domain Dedication waiver (http://creativecommons.org/publicdomain/zero/1.0/) applies to the data made available in this article, unless otherwise stated in a credit line to the data. 
elongation cycle, $\mathrm{C} 16$ and $\mathrm{C} 18$ acyl-CoA precursors. VLCFA derivatives are produced in two ways: most wax compounds, including aldehydes, alkanes, secondary alcohols, and ketones, are biosynthesized via the alkaneforming pathway, whereas alcohols and esters are biosynthesized via the alcohol-forming pathway [6].

In Arabidopsis thaliana, many wax-biosynthesis genes have been identified, including the FAE complex genes KCS1, KCS2, KCS6, KCS9, KCS2O and KCR1 [7, 8]; the VLCFA modification genes CER4, MAH1, CER1, CER3, CER2 (-like) and CER26 [9-11]; the transporter genes DESPERADO/AtWBC11, ABCG transporters, and the $C E R 5$ gene $[12,13]$; and the regulatory genes WIN1/ SHN1, SHN2, SHN3, MYB30, MYB41, CFL1, CER7, and CER9 [14, 15]. Mutants of these wax-related genes show a decrease or lack of cuticular waxes, resulting in glossy phenotypes.

Among the wax-deficient mutants, cer2 shows glossy green appearance in stems and siliques due to the decrease of all waxes longer than C28 [16-18]. CER2 gene has been mapped to At4g24510 via chromosome walking and T-DNA insertion identification, which is expressed in aerial plant tissues, with higher level in stems and siliques, and trace level in leaves [17, 18]. CER2 encodes an endoplasmic reticulum-localized protein homologous to BAHD acyltransferase, responsible for elongation of VLCFAs longer than C28, but unlike a typical BAHD acyltransferase, the CER2 protein localize exclusively to the nucleus, and the catalytic acyltransferase motif was not conserved and not required for its function [10].

Mutants showing glossy phenotypes have also been identified in various Brassica species, including B.oleracea [19-22], B. napus [23], and B. rapa [24]. In cabbage, 3 glossy mutants have been reported. Liu et al. identified a dominant glossy green cabbage mutant, 10Q-974gl, and mapped the target gene BoGL1 to C08, between marker SSRC08-76 (41.51 $\mathrm{Mb}$ ) and the end of the chromosome; two tandem genes, Bol018503 and Bol018504, which are homologous to CER1 in Arabidopsis, were determined to be candidates for BoGL1 [20]. Liu et al. reported a recessive glossy green cabbage mutant, 10Q-961, and mapped the target gene Cgl1 to a 188.7 $\mathrm{kb}$ interval at the end of C08; interestingly, CER1 was also predicted as the candidate [21]. Gene editing by CRISPR/ Cas9 systems further confirmed the essential role of BoCER1 in synthesis of cuticular wax [25]. Liu et al. reported other recessive cabbage mutants (HUAYOU2 and LD1OGL) and identified Bol013612 (which is homologous to CER4) as the candidate [19, 21]. The ectopic expression of Bol013612 in Arabidopsis rescued the wax deficiency of cer4 mutants [19].

Broccoli (Brassica oleracea L. var. italica), a subspecies of $B$. oleracea, is an important vegetable crop widely cultivated worldwide. Previously, researchers have reported glossy mutants of broccoli [26, 27], but no genetic studies on these plants have been conducted. In this study, we identified a novel spontaneous glossy mutant B156, of broccoli. Using an $\mathrm{F}_{2}$ population, we mapped the target gene BoGL5 to a $94 \mathrm{~kb}$. Subsequent gene prediction, sequencing and functional analyses suggested that $B o C E R 2$ was a highly plausible candidate gene for BoGL5.

\section{Results \\ Phenotype and inheritance of the glossy traits of the B156 plants}

In comparison with the glaucous appearance of wildtype plants B3 (Fig. 1D and E), the outer surfaces of B156, including the stems, leaves and siliques, showed glossy traits (Fig. 1A and B), possibly due to reduced load of cuticular waxes. Its consumed production, i.e. the flowering inflorescence (head and stalk) also became glossy green (Fig. S1). Other than the glossy appearance, the mutant showed no difference to wild type plants. The glossy phenotype of the B156 plants resembled the reported cabbage glossy mutants 10Q-974gl, HUAYOU2 and $L D 10 G L[19,21]$, but unlike that of $10 Q-974 g l$, its male fertility was not changed.

Observation by scanning electron microscopy (SEM) revealed that B3 leaves were covered with rod-shaped wax crystals, whereas B156 leaves were nearly devoid of wax crystals (Fig. 1C and F). Analysis of cuticular wax load and composition by GC-MS revealed a $63.5 \%$ reduction in total wax load of B156 mutant. All waxes longer than C28, except for C29 aldehydes, were dramatically decreased; in contrast C26, C27 and C28 waxes were slightly increased (Fig. 1G), indicating elongation of C28 VLCFA was blocked in B156 mutant.

\section{Fine mapping of the BoGL5 gene}

B156 was crossed with B3, a wild-type broccoli inbred line, and all the derived $F_{1}$ plants showed a normal phenotype. The $F_{2}$ plants segregated as 3:1 (1296 normal plants:412 glossy plants; $X^{2}=0.70<\chi_{0.05,1}^{2}=3.84$ ) according to a $X^{2}$ test, indicating that this glossy trait is controlled by a single recessive gene, BoGL5.

Primarily, ninety-three InDel primers covering all 9 chromosomes were designed to detect polymorphisms between the parents (B156 and B3) and the two DNA pools (the G pool and NG pool) (Table S1). A total of 61 primers showed polymorphisms between the parents, but only three markers, Broc2 (C01: 8,156,135), Broc3 (C01: 13,116,351) and Broc4 (C01: 18,102,957), were polymorphic between the two DNA pools (Table S1). A total of 89 randomly selected glossy $F_{2}$ individuals were used to confirm the linkage and estimate the genetic distances. Broc2 and Broc3 were upstream of BoGL5, with genetic distances of $7.8 \mathrm{cM}$ and $1.1 \mathrm{cM}$, respectively; Broc4 was downstream of BoGL5, with a genetic distance of $2.2 \mathrm{cM}$. 


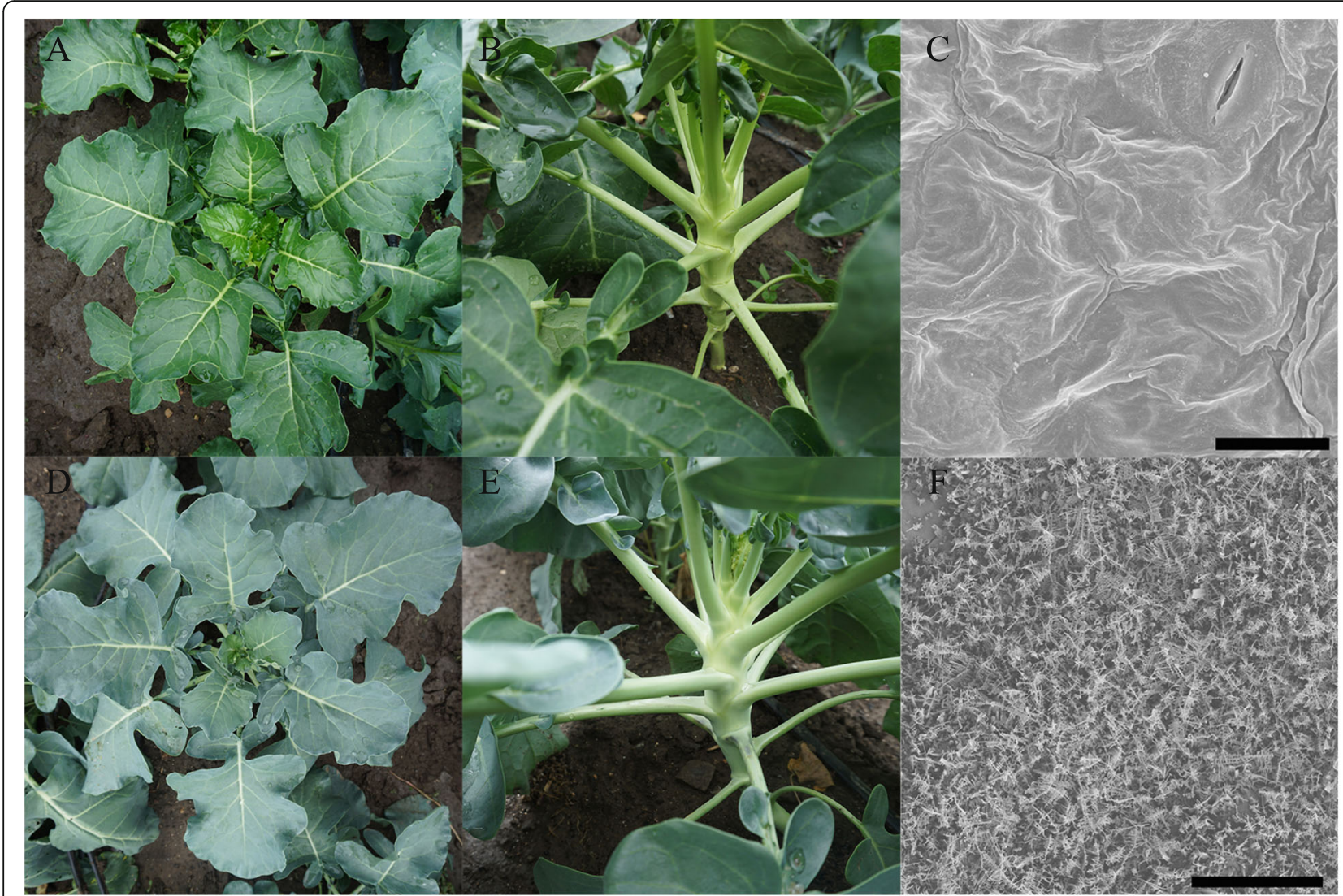

G

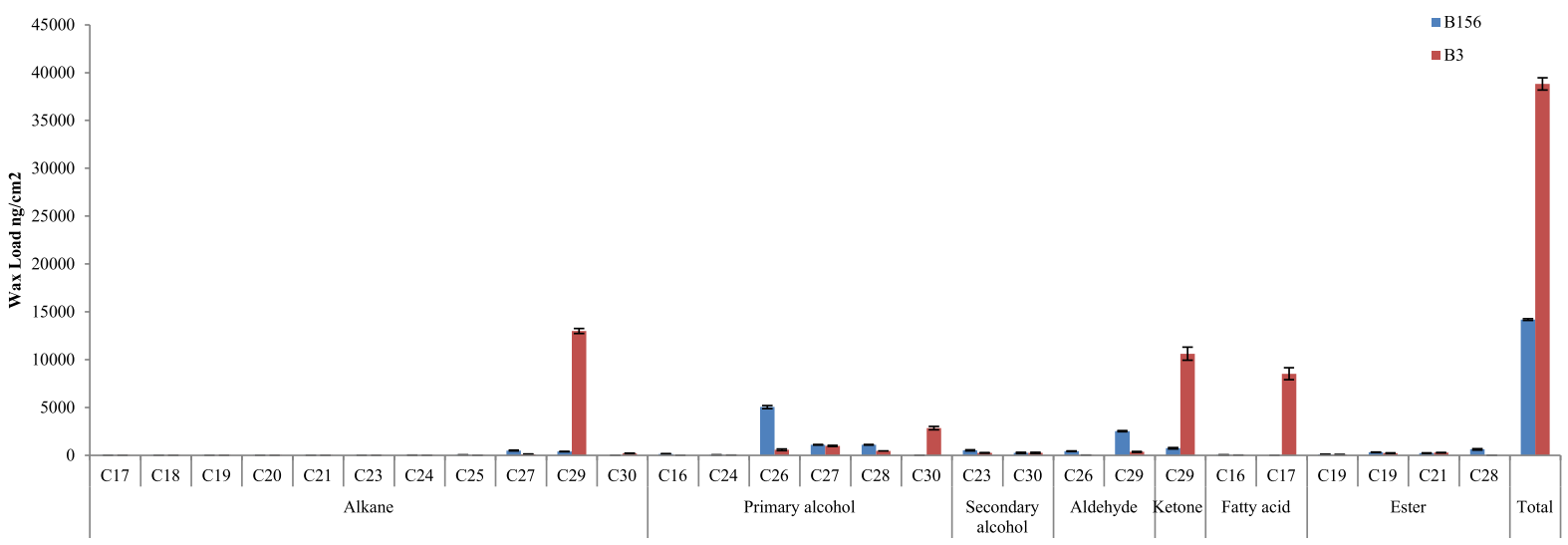

Fig. 1 Phenotypes and wax loads of the mutant B156 and the wild-type B3 plants. B156 showed glossy appearance (a, b) in contrast with glaucous appearance of B3 (d, e). Scanning electron microscopy revealed dramatically reduced waxes on leaf of B156 (c) comparing with B3 (f), bar = $150 \mu$ m. Chromatography-mass spectrometry revealed total waxes and most > C28 components were significantly decreased in B156 (g)

To narrow the candidate mapping region, 32 InDel markers within the $10 \mathrm{Mb}$ genomic region were designed between Broc2 and Broc4. Ultimately, we obtained 21 markers linked to BoGL5. Two markers, Broc94 (C01: 11,005,498) and Broc4 (C01: 18,102,957), were used to genotype all 412 glossy $F_{2}$ individuals to detect recombinants. As a result, we detected 20 recombinant individuals for Broc94 and 46 for Broc4 (Table S2). All recombinant individuals were further genotyped using eight markers located between Broc94 and Broc4 (Table S2). Ultimately, we mapped BoGL5 to a $94.1 \mathrm{~kb}$ genomic region flanked by markers Broc123 and Broc117, each with a genetic distance of $0.12 \mathrm{cM}$. A genetic map is shown in Fig. 2A. 
BolC1t01909H (BoCER2) is a strong candidate for BoGL5 According to the gene prediction information in the HDEM reference genome, fifteen genes were located within the $94.1 \mathrm{~kb}$ mapping region (Table 1 ). Considering the cuticular waxes defects of B156, the causal gene should be structural, transporter or regulator gene associated with biosynthesis of VLCFAs and their derivatives. Among the fifteen genes, only one named BolC1t01909H was related to the biosynthesis of plant cuticular waxes. BolC1t01909H is predicted to encode an HXXXD-type acyltransferase family protein required for C28-to-C30 fatty acid elongation, consistent with the blocked elongation of C28 VLCFA and 63.5\% total wax load reduction in B156 mutant. Additionally, BolC1t01909H showed nucleotide variation and differential expression levels between B156 and B3 plants (see the following results). Based on information of gene annotations, wax loads, gene sequencing and relative expression level results, we tentatively designated BolC1t01909H (BoCER2) as the candidate gene for BoGL5.

\section{Bocer2 of B156 mutant harbored a SNP that disrupted a conserved site adjacent to the HXXXD motif}

We retrieved the gene sequence of BolC1t01909H (BoCER2) from the HDEM reference genome, the transcript structure of which seemed to be wrongly predicated. The full-length transcript of BolC1t01909H was obtained from an unpublished PacBio-based [PacBio RSII (Pacific Biosciences, Menlo Park, CA, USA)] RNA- seq dataset and amplified from B156 and B3 plants. The results revealed that the coding sequence of BoCER2 is $1254 \mathrm{nt}$ in length and comprised two exons (Fig. 2B). The deduced BoCER2 protein shared $74 \%$ identity with Arabidopsis CER2.

To determine whether BoCER2 had nucleotide variation between $\mathrm{B} 156$ and $\mathrm{B} 3$, the $2 \mathrm{~kb}$ promoter region and $2462 \mathrm{bp}$ gene body region were amplified from the genomic DNA of B156 and B3 plants. The BoCER2 transcript was amplified from the cDNA of B156 and B3. There was no nucleotide variation detected within the promoter region, whereas in the coding region, only one SNP mutation ( $G$ to $T$ ) was detected in B156, located within the second exon, $1693 \mathrm{bp}$ downstream of the start codon, corresponding to the 485th nucleotide in the CDS (Fig. 2B). This mutation resulted in a W-to-L transition at the 162nd amino acid of the deduced protein sequence. Protein sequence analyses using BoCER2 and its close relatives revealed that the mutation site was adjacent to the HXXXD motif and was conserved in these species (Fig. 3). These results suggested that the 485th mutation might disrupt the function of BoCER2 and might be responsible for the glossy trait of B156.

\section{Expression analysis of BoCER2}

We performed qRT-PCR to reveal the expression pattern of BoCER2 in wild-type B3 and mutant B156 plants. The results showed that BoCER2 was expressed in the stems, leaves and siliques but was not expressed in the
$\mathbf{A}$

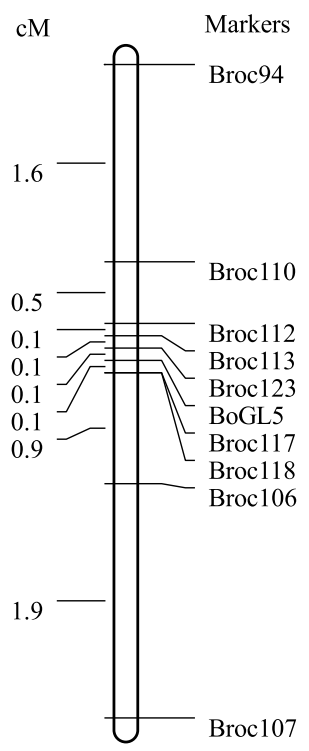

B

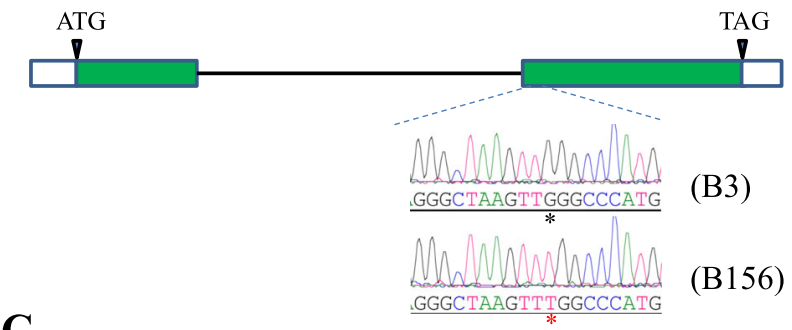

C

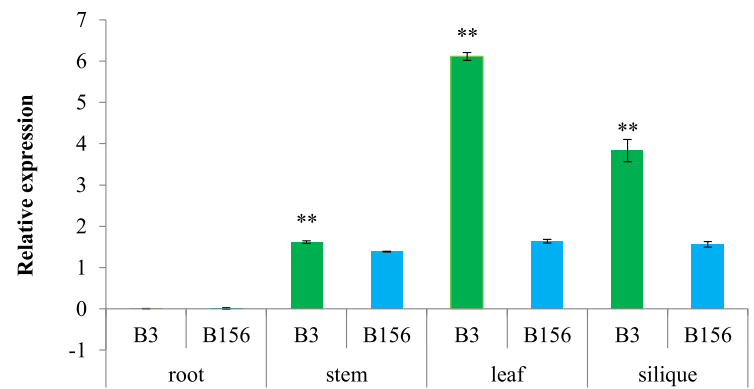

Fig. 2 Linkage map and candidate gene analyses. BoGL5 was mapped to an interval between Broc123 and Broc117 on chromosome C01 (a). Gene structure and allelic variation of the candidate gene BoCER2 (b). BoCER2 was significantly downregulated in the stems, leaves and siliques of B156 compared with those of B3 (c) 
Table 1 Predicted genes in the target genomic region

\begin{tabular}{lllll}
\hline Gene ID & Start position & Stop position & Homologous gene in A. thaliana & Annotation \\
\hline BolC1t01905H & $12,808,460$ & $12,810,897$ & AT4G24460 & CRT (chloroquine-resistance transporter)-like transporter 2 \\
BolC1t01906H & $12,811,839$ & $12,813,742$ & AT4G24470 & GATA-type zinc finger protein with a TIFY domain \\
BolC1t01907H & $12,835,665$ & $12,839,913$ & AT4G24480 & Protein kinase superfamily protein \\
BolC1t01908H & $12,841,073$ & $12,845,010$ & AT4G24490 & Hydroxyproline-rich glycoprotein family protein \\
BolC1t01909H & $12,845,188$ & $12,849,854$ & AT4G24510 & HXXXD-type acyltransferase family protein \\
BolC1t01910H & $12,856,294$ & $12,860,159$ & AT4G24520 & P450 reductase 1 \\
BolC1t01911H & $12,861,674$ & $12,865,576$ & - & NADPH-cytochrome P450 reductase 1-like \\
BolC1t01912H & $12,866,709$ & $12,870,028$ & AT4G24530 & O-fucosyltransferase family protein \\
BolC1t01913H & $12,870,641$ & $12,873,198$ & AT4G24540 & AGAMOUS-like 24 \\
BolC1t01914H & $12,874,770$ & $12,875,093$ & - & Polyadenylate-binding protein-interacting protein 6-like \\
BolC1t01915H & $12,878,887$ & $12,879,195$ & - & Profilin-1-like \\
BolC1t01916H & $12,886,194$ & $12,889,176$ & AT4G24550 & Clathrin adaptor complexes medium subunit family protein \\
BolC1t01917H & $12,889,708$ & $12,894,099$ & AT4G24560 & ubiquitin-specific protease 16 \\
BolC1t01918H & $12,899,929$ & $12,900,876$ & AT4G24570 & dicarboxylate carrier 2 \\
BolC1t01919H & $12,901,237$ & $12,907,174$ & AT4G24580 & Rho GTPase activation protein (RhoGAP) with a PH domain
\end{tabular}

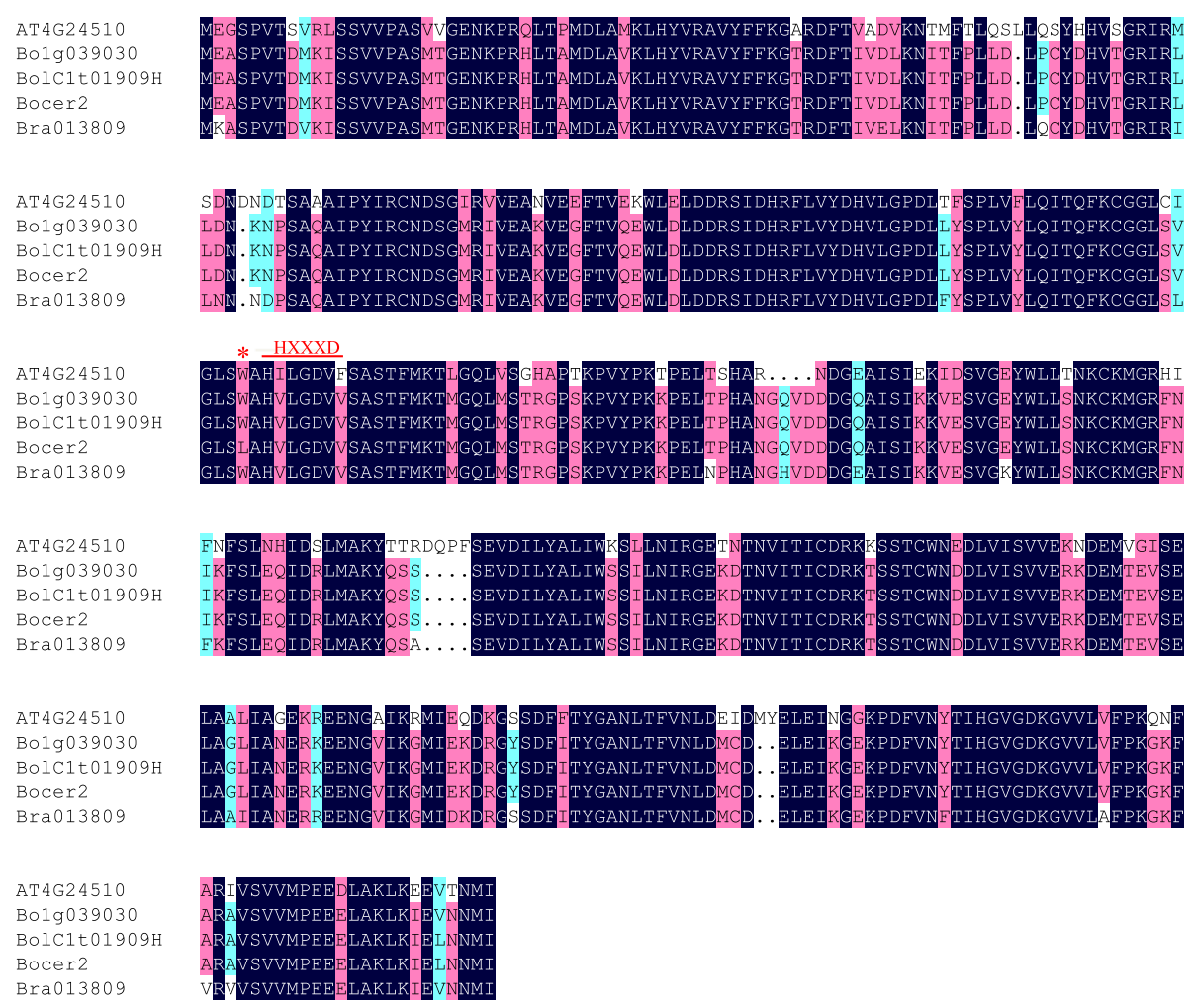

Fig. 3 Protein sequence alignment of BoCER2 and its relatives. AT4G24510, CER2 of A. thaliana; Bo1g039010, BoCER2 in TO1000 (Chinese kale); BolC1t01909H, BoCER2 in B3 and HDEM (broccoli); Bocer2, mutant-type BoCER2 in B156; Bra013809, BraCER2 of B. rapa. The 162nd amino acid is indicated by the red asterisk 
roots (Fig. 2C). By comparing the expression levels of $B o C E R 2$ between $\mathrm{B} 3$ and $\mathrm{B} 156$. The results showed that the expression of BoCER2 was significantly downregulated in the leaves, stems and siliques of the B156 mutant (Fig. 2C).

\section{Ectopic expression of BoCER2 rescued the phenotype of Arabidopsis cer2 mutant}

We failed to introduce the BoCER2 from B3 to the B156 mutant, because this plant material was recalcitrant to Agrobacterium-mediated transformation. Therefore, the function of BoCER2 was tested in A. thaliana cer2 mutant. We obtained 16 BoCER2-overexpression lines and 14 Bocer2-overexpression lines in the background of Arabidopsis cer2 mutant. The phenotype and wax load of BoCER2-overexpression lines restored to wild type levels, whereas the Bocer2-overexpression still showed glossy appearance and lacking of cuticular waxes (Figs. 4 and 5), indicating that the broccoli BoCER2 is a functional gene responsible for wax biosynthesis, and Bocer2 is a loss-of-function allele.

\section{Discussion}

The surfaces of terrestrial plants are covered by cuticles providing them with glaucous appearance. In contrast to

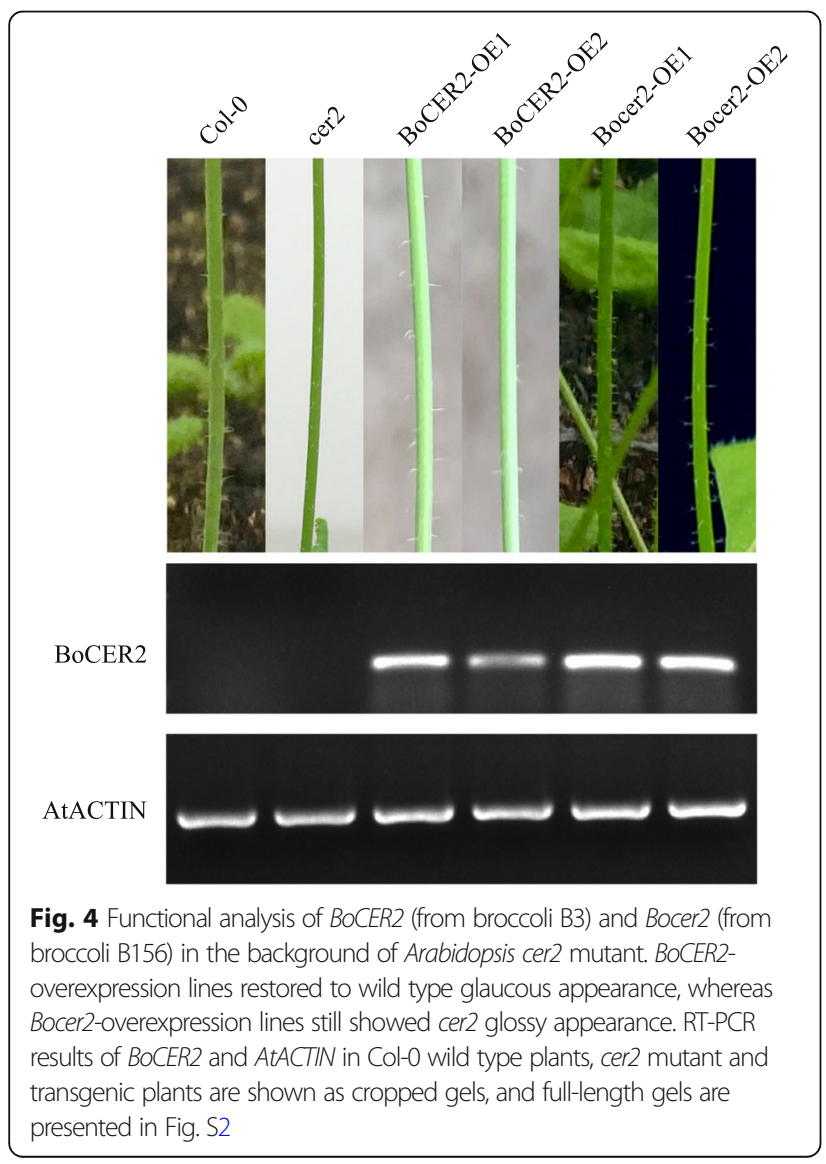

wild-type plants, many mutants with a glossy appearance and their corresponding genes have been identified in $A$. thaliana, Maize and rice, revealing defects in cuticular wax biosynthesis. In addition to these model plant species, some glossy mutants have also been identified in Brassica species, including cabbage [19-22], Chinese cabbage [23], and rapeseed [24]. In this study, we identified a novel broccoli mutant, B156, whose glossy phenotype was similar to that of previously reported $10 \mathrm{Q}$ 974gl, 10Q-961, HUAYOU2 and LD10GL cabbage lines [19-22]. Fine mapping, gene sequencing, gene expression and functional analyses revealed that BoCER2 was a candidate gene responsible for the glossy trait.

Although the HXXXD acyltransferase motif was previously reported not required for CER2 function [10], this study indicated that the conserved sequence around HXXXD motif was essential for the function of BoCER2. Arabidopsis CER2 was identified as At4g24510 [16-18], which encodes an endoplasmic reticulum-localized protein homologous to BAHD acyltransferase. Its biochemical function in C28 VLCFA elongation was indicated by an assay in yeast [10]. However, the catalytic acyltransferase motif of CER2 was not conserved and not required for function, which is very different from the catalytic mechanism of other members of the BAHD family [10]. In this study, a SNP mutation within BoCER2 in B156 should result in a W-to-L transition at the 162nd amino acid of the deduced BoCER2 protein sequence, which might disrupt the function of BoCER2 and result in the glossy traits of B156. Sequence comparison and ectopic expression in Arabidopsis revealed that the 162nd amino acid is a conserved site essential for the function of BoCER2. Thus, B156 should be a useful material for further characterization of the catalytic mechanism of CER2-related proteins.

We found that broccoli BoCER2 might have experienced functional and expression divergence in comparison with Arabidopsis CER2. The C29 aldehydes are increased in B156 mutant, which is opposite to Arabidopsis cer 2 mutant, indicating that BoCER2 may not be responsible for VLCFA elongation of aldehydes. The expression divergence between the BoCER2 and Arabidopsis CER2 is obvious. Arabidopsis CER2 is expressed in aerial plant tissues, with high levels in stems and siliques, and trace level in leaves, but not in roots $[17,18]$. Its mutant cer 2 showed significantly decreased total wax load on the stem $[10,17,18]$. However, the composition and load of wax on the leaves had no difference, possibly due to the functional redundancy of CER2, CER2-LIKE1 and CER2-LIKE2 in the leaves [10]. BoCER2 was expressed in aerial organs similar to that of Arabidopsis CER2. However, the BoCER2 transcript was highest abundant in the leaves. Interestingly, in contrast to the Arabidopsis cer 2 mutant, the B156 mutant showed an 


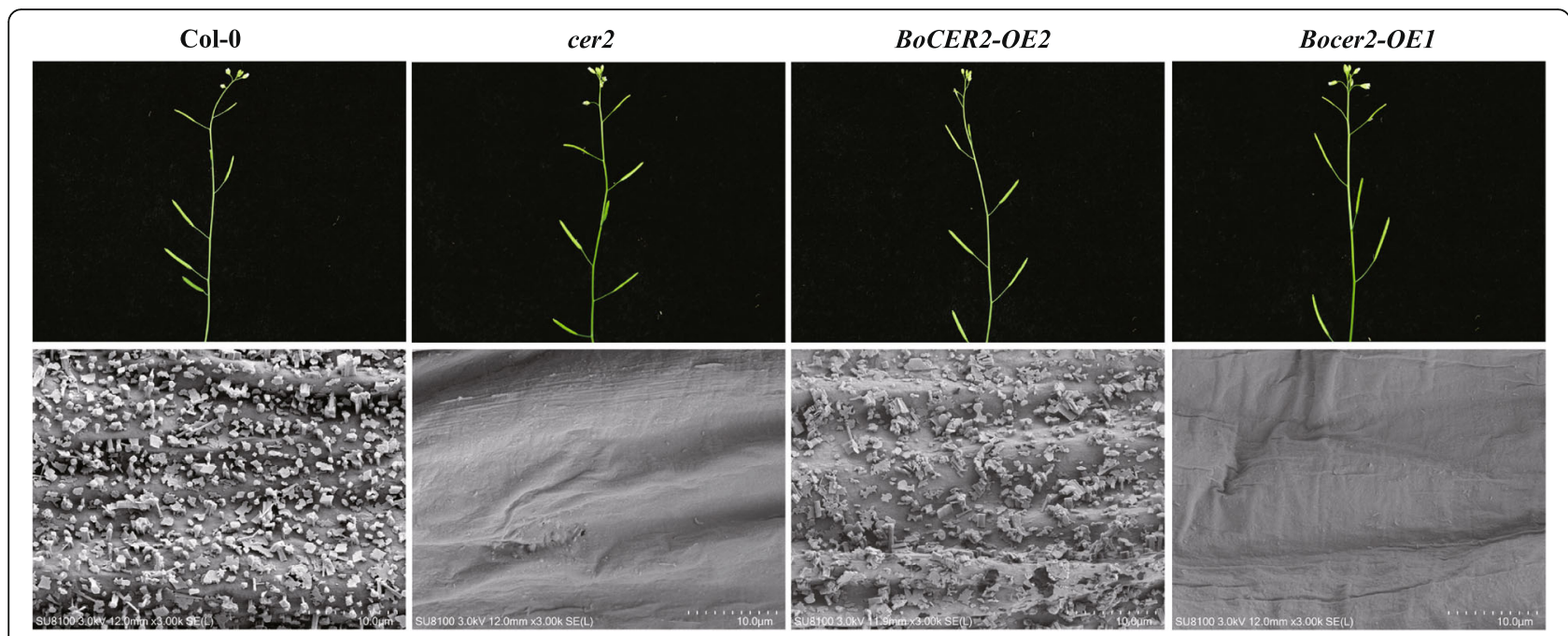

Fig. 5 Phenotype of Arabidopsis stems and siliques, and scanning electron microscopy images of wax crystals on the surface of Arabidopsis stems. The Col-0 wild type and BoCER2-OE1 plants are covered with wax crystals. The cer2 mutant and Bocer2-OE1 plants lack wax crystals

obvious glossy appearance of its leaves, indicating the predominant role of BoCER2 in waxes accumulation on the surface of leaves. Further studies on the homologies among CER2, CER2-LIKE1 and CER2-LIKE2 in broccoli should provide new insight into the expression and functional divergence of these genes and leaf wax biosynthesis in B. oleracea.

The B156 mutant and the cloned BoCER2 have significance in breeding $B$. oleracea varieties. The glossy green broccoli mutant is, although currently not preferred by customers at least in China, useful to cabbage, another subspecies of $B$. oleracea, because the leaf product of this type is fresh and tender, and thus preferred by some customers. So breeders can introduced the locus from B156 to cabbage, which would help to create new glossy green varieties without any penalties of male fertility (unlike the Bocer1 cabbage mutant). Moreover, identification genes involved in cuticular wax biosynthesis is of significance because of their roles in protecting plants against biotic and abiotic stresses. We have proved $B o C E R 2$ to be a functional gene responsible for cuticular wax biosynthesis. It can be chosen as a target, which can be enhanced for better resistance to biotic and abiotic stresses, or knocked out by CRISPR/Cas9 system for direct creation of glossy green materials.

\section{Conclusions}

In summary, we conducted phenotype analyses and genetic mapping of a novel glossy green mutant B156 in broccoli. Comparing with the wild type line, B156 mutant showed $63.5 \%$ reduction in total wax load, and lacking waxes mostly longer than $\mathrm{C} 28$, indicating elongation of C28 VLCFA was blocked in this mutant. Inheritance analysis revealed that the glossy trait was controlled by a recessive locus that was mapped to a $94.1 \mathrm{~kb}$ interval on C01 chromosome. In the target interval, only BolC1t01909H (BoCER2) was identified as a candidate for BoGL5. Bocer2 of B156 mutant harbored a key SNP mutation located in a conserved site, providing a useful material for further characterization of the catalytic mechanism of CER2-related proteins. Further function characterization in A. thaliana cer2 mutant suggested that BoCER2 is a functional gene responsible for wax biosynthesis, and Bocer2 is a loss-of-function allele. This study lays a foundation for further characterization of $B o C E R 2$ and its utilization in enhancing stress tolerance and breeding of special cultivars with glossy appearance in B. oleracea.

\section{Methods}

\section{Plant materials}

B156 is a spontaneous glossy broccoli mutant generated from a natural broccoli population in 2010 and maintained by selfing. Another inbred line, B3, was crossed with a $B 156$ parent to produce $F_{1}$ plants. An $F_{2}$ population comprising 1708 individuals was generated from self-pollination of the $\mathrm{F}_{1}$ progeny. Arabidopsis Col-0 wild-type line was preserved in our research group, and cer2 mutant (SALK_084443C) was ordered from AraShare (https://www.arashare.cn/). All the plant materials were owned by the Institute of Vegetables and Flowers, Chinese Academy of Agriculture Sciences (IVFCAAS, Beijing, China).

\section{Cuticular waxes loads and compositions detection by SEM and GC-MS}

Fresh leaves were collected from 8-week-old plants of B3 and B156. Scanning electron microscopy (SEM) and 
gas chromatography-mass spectrometry (GC-MS) were conducted as described by Liu et al. [19] and Cao et al. [25]. The wax loads were the means of three repetitions as $\mathrm{ng} / \mathrm{cm} 2$. Additionally, stems of Arabidopsis thaliana were collected from 4-week-old plants for SEM analyses.

\section{Whole-genome nucleotide variation calling and primer design}

We performed whole-genome resequencing of the parents, B156 and B3, using the Illumina HiSeq 2500 platform (Illumina, Inc., San Diego, CA, USA). The resequencing data were mapped to the reference genome sequence of broccoli "HDEM" (http://www.genoscope.cns.fr/externe/plants/index. html) [28], and single-nucleotide polymorphism (SNP) and InDel variations between the parents were retrieved. According to a previous study [29], InDel primers evenly distributed across the 9 chromosomes were designed using Primer 3.

\section{DNA isolation and fine mapping of the BoGL5 gene}

Genomic DNA was extracted from young leaves using a modified cetyltrimethylammonium bromide (CTAB) protocol [30]. All primers were screened using the parents' DNA as a template. Polymorphic markers between the parents were further screened by bulked segregant analysis (BSA) [30]. Generally, two DNA pools (10 glossy $F_{2}$ individuals for the $G$ pool and 10 nonglossy $F_{2}$ individuals for the NG pool) were constructed to detect markers linked to BoGL5. More InDel primers were designed around the linked markers to search for more linked markers. All glossy $\mathrm{F}_{2}$ individuals were genotyped to detect recombinants, construct a linkage map and narrow the location of the target gene. Polymerase chain reaction (PCR) and polyacrylamide gel electrophoresis (PAGE) were performed according to the methods in a previous report [31].

\section{RNA isolation, CDNA synthesis and expression analysis of the candidate gene by real-time PCR}

Total RNA was extracted from tissues of the roots, stems, leaves, and siliques of B156 and B3 plants using an RNAprep Pure Plant Kit (Tiangen, Beijing, China), and first-strand cDNA was synthesized using a PrimeScript 1st Strand cDNA Synthesis Kit (Tiangen, Beijing, China). The protocols were performed according to the manufacturer's instructions.

Quantitative RT-PCR (qRT-PCR) was performed to compare the expression levels of BoCER2 in the B3 and B156 tissues. The qRT-PCR mixture was prepared in conjunction with SYBR Premix Ex Taq II (Tli RNase H Plus; Takara, Dalian, China), and the amplification was performed on a CFX96 Touch Real-Time PCR Detection System (Bio-Rad, Hercules, CA, USA). All samples were assayed in triplicate.
The transcript abundance of BoCER2 in the samples was calculated using the $2^{-\triangle \Delta C T}$ method. The specific primers for BoCER2 and B. oleracea actin are listed in Table S3.

\section{Gene amplification and sequence analyses of BoCER2}

The reference sequence of $B o C E R 2$ was acquired from the HDEM reference genome and unpublished RNA-seq data. Based on the reference sequence, primers were designed using Primer 5.0 software. The $\sim 2 \mathrm{~kb}$ promoter and gene body region was amplified using genomic DNA and/or first-strand cDNA of B156 and B3 as templates. PCR was performed using Q5 High-Fidelity DNA Polymerase (NEB).

Sequences of $A$. thaliana CER2 were downloaded from The Arabidopsis Information Resource (TAIR) database (https://www.arabidopsis.org/). BLASTP was used to query the deduced amino acid sequence of BoCER2 for its relatives in B. rapa and B. napus. The obtained proteins were aligned using DNAMAN 6.0 (Lynnon Biosoft, CA, San Ramon, USA).

\section{Plasmid construction and Arabidopsis transformation} The coding sequences of BoCER2 and Bocer 2 were amplified from 1st Strand cDNA of B3 and B156 respectively. The purified PCR products were inserted into a modified binary vector $\mathrm{pBWA}(\mathrm{V}) \mathrm{BS}$ (reconstructed from pCAMBIA1301) [32], placed downstream of the constitutive $35 \mathrm{~S}$ promoter. The constructs were transformed into competent Agrobacterium tumefaciens GV3101, and into Arabidopsis cer2 mutant (SALK_084443C) using the floral dip method [33]. Seeds were screened for hygromycin resistance on MS (Murashige and Skoog) medium containing $30 \mathrm{mg} / \mathrm{L}$ of hygromycin, and were further confirmed by RT-PCR analysis.

\section{Abbreviations \\ SEM: Scanning electron microscopy; GC-MS: Chromatography-mass spectrometry; VLCFAs: Very long chain fatty acids; ER: Endoplasmic reticulum; FAE: Multienzymatic fatty acid elongase; CRISPR: Clustered regularly interspaced short palindromic repeats; SNP: Single-nucleotide polymorphism; InDel: Insertion or deletion polymorphism; qRT-PCR: Quantitative real-time polymerase chain reaction; BSA: Bulked segregant analysis; \\ PAGE: Polyacrylamide gel electrophoresis; CTAB: Cetyltrimethylammonium bromide}

\section{Supplementary Information}

The online version contains supplementary material available at https://doi. org/10.1186/s12864-021-08143-7

Additional file 1: Table S1. Information of location, sequence, genotype (identified according to the resequencing data), and polymorphisms of all the markers used for genetic mapping.

Additional file 2: Table S2. Genotypic and phenotypic data of the $F_{2}$ population. The plants were firstly genotyped by Broc94 and Broc4 to detect recombinants, which were further genotyped by additional eight markers.

Additional file 3: Table S3. Primers used in this study for genetic mapping, gene amplification and RT-PCR. 
Additional file 4: Figure S1. Field performance of B3, B156 and the $F_{2}$ population generated from the parents.

Additional file 5: Figure S2. Full-length gels showing the RT-PCR results of BoCER2 and AtActin. The corresponding cropped gels were shown in Fig. 4.

\section{Acknowledgments}

We thank Prof. Xia Cui and Dr. Haijing Wang for experimental assistance from Institute of Vegetables and Flowers, Chinese Academy of Agricultural Sciences.

\section{Authors' contributions}

$\mathrm{FH}, \mathrm{JH}$ and $\mathrm{ZL}$ designed and performed the experiments. HF and $\mathrm{ZL}$ wrote and revised the paper. QX, LY, MZ, YZ, HL, YW, ZF and JJ coordinated and designed the study. All authors have read and approved the final manuscript.

\section{Funding}

This work was supported by grants from the Central Public-interest Scientific Institution Basal Research Fund (Grant No. IVFBRF2020003; IVF-BRF2021003), the National Natural Science Foundation of China (Grant No. 31501761), the China Agriculture Research System (Grant No. CARS-23-05A), the Agricultural Science and Technology Innovation Program (ASTIP), and the State Key Laboratory of VegetableGermplasm Innovation (Grant No. SKL-VGI).

\section{Availability of data and materials}

The raw sequencing data in this study are available in the SRA database (BioProject accession number: PRJNA695138). These data can be accessed using following link: https://www.ncbi.nlm.nih.gov/bioproject/PRJNA695138.

\section{Declarations}

Ethics approval and consent to participate

We confirm that all experimental protocols were approved by Ethics Committee of the Institute of Vegetables and Flowers, Chinese Academy of Agriculture Sciences (IVFCAAS, Beijing, China). The utilization of all plant materials in this study complies with relevant institutional and national guidelines and legislation.

\section{Consent for publication}

Not applicable.

\section{Competing interests}

The authors declare that they have no competing interests.

Received: 18 April 2021 Accepted: 1 November 2021

Published online: 10 November 2021

\section{References}

1. Shepherd T, Wynne GD. The effects of stress on plant cuticular waxes. New Phytol. 2006;171(3):469-99. https://doi.org/10.1111/j.1469-8137.2006.01826.x.

2. Beisson F, Li-Beisson Y, Pollard M. Solving the puzzles of cutin and suberin polymer biosynthesis. Curr Opin Plant Biol. 2012;15(3):329-37. https://doi. org/10.1016/j.pbi.2012.03.003.

3. Müller C. Plant-Insect interactions on cuticular surfaces. Annu Plant Rev Biol Plant Cuticle. eds M. Riederer and C. Müller (Oxford: Blackwell Publishing Ltd.). 2006;23:398-422

4. Samuels $L$, Kunst $L$, Jetter $R$. Sealing plant surfaces: cuticular wax formation by epidermal cells. Plant Biol. 2008;59(1):683-707. https://doi.org/10.1146/a nnurev.arplant.59.103006.093219.

5. Kunst L, Samuels L. Plant cuticles shine: advances in wax biosynthesis and export. Curr Opin Plant Biol. 2009;12(6):721-7. https://doi.org/10.1016/j.pbi.2 009.09.009.

6. Rowland O, Zheng H, Hepworth SR, Lam P, Jetter R, Kunst L. CER4 encodes an alcohol-forming fatty acyl-coenzyme a reductase involved in cuticular wax production in Arabidopsis. Plant Physiol. 2006;142(3):866-77. https://doi. org/10.1104/pp.106.086785.

7. Franke R, Höfer R, Briesen I, Emsermann M, Efremova N, Yephremov A, et al. The DAISY gene from Arabidopsis encodes a fatty acid elongase condensing enzyme involved in the biosynthesis of aliphatic suberin in roots and the chalaza-micropyle region of seeds. Plant J. 2009;57(1):80-95. https://doi.org/10.1111/j.1365-313X.2008.03674.x.

8. Kim J, Jung JH, Lee SB, Go YS, Kim HJ, Cahoon R, et al. Arabidopsis 3-ketoacylcoenzyme a synthase 9 is involved in the synthesis of tetracosanoic acids as precursors of cuticular waxes, suberins, sphingolipids, and phospholipids. Plant Physiol. 2013;162(2):567-80. https://doi.org/10.1104/pp.112.210450.

9. Li F, Wu X, Lam P, Bird D, Zheng H, Samuels L, et al. Identification of the wax ester synthase/acyl-coenzyme a: diacylglycerol acyltransferase WSD1 required for stem wax ester biosynthesis in Arabidopsis. Plant Physiol. 2008; 148(1):97-107. https://doi.org/10.1104/pp.108.123471.

10. Haslam TM, Manas-Fernandez A, Zhao L, Kunst L. Arabidopsis ECERIFERUM2 is a component of the fatty acid elongation machinery required for fatty acid extension to exceptional lengths. Plant Physiol. 2012;160(3):1164-74. https://doi.org/10.1104/pp.112.201640

11. Pascal S, Bernard A, Sorel M, Pervent M, Vile D, Haslam RP, et al. The Arabidopsis cer26 mutant, like the cer2 mutant, is specifically affected in the very long chain fatty acid elongation process. Plant J. 2013;73(5):733-46. https://doi.org/10.1111/tpj.12060.

12. Bird D, Beisson F, Brigham A, Shin J, Greer S, Jetter $R$, et al. Characterization of Arabidopsis ABCG11/WBC11, an ATP binding cassette (ABC) transporter that is required for cuticular lipid secretion. Plant J. 2007:52(3):485-98. https://doi.org/10.1111/j.1365-313X.2007.03252.x.

13. Panikashvili D, Savaldigoldstein S, Mandel T, Yifhar T, Franke RB, Höfer R, et al. The Arabidopsis DESPERADO/AtWBC11 transporter is required for cutin and wax secretion. Plant Physiol. 2007;145(4):1345-60. https://doi.org/1 0.1104/pp.107.105676

14. Lee SB, Suh MC. Recent advances in cuticular wax biosynthesis and its regulation in Arabidopsis. Mol Plant. 2013;6(2):246-9. https://doi.org/10.1 093/mp/sss159.

15. McGlew K, Shaw V, Zhang M, Kim RJ, Yang W, Shorrosh B, et al. An annotated database of Arabidopsis mutants of acyl lipid metabolism. Plant Cell Rep. 2015;34(4):519-32. https://doi.org/10.1007/s00299-014-1710-8.

16. Jenks MA, Tuttle HA, Eigenbrode SD, Feldmann KA. Leaf epicuticular waxes of the eceriferum mutants in Arabidopsis. Plant Physiol. 1995;108(1):369-77. https://doi.org/10.1104/pp.108.1.369.

17. Negruk V, Yang P, Subramanian M, McNevin JP, Lemieux B. Molecular cloning and characterization of the CER2 gene of Arabidopsis thaliana. Plant J. 1996:9(2):137-45. https://doi.org/10.1046/j.1365-313X.1996.09020137.x.

18. Xia Y, Nikolau BJ, Schnable PS. Cloning and characterization of CER2, an Arabidopsis gene that affects cuticular wax accumulation. Plant Cell. 1996; 8(8):1291-304. https://doi.org/10.1105/tpc.8.8.1291.

19. Liu D, Tang J, Liu Z, Dong X, Zhuang M, Zhang Y, et al. Cgl2 plays an essential role in cuticular wax biosynthesis in cabbage (Brassica oleracea L. var. capitata). BMC Plant Biol. 2017;17(1):223

20. Liu D, Tang J, Liu Z, Dong X, Zhuang M, Zhang Y, et al. Fine mapping of BoGL1, a gene controlling the glossy green trait in cabbage (Brassica oleracea L. Var. capitata). Mol Breed. 2017;37(5):69.

21. Liu Z, Fang Z, Zhuang M, Zhang Y, Lv H, Liu Y, et al. Fine mapping and analysis of Cgl1, a gene conferring glossy trait in cabbage (Brassica oleracea L. var. capitata). Front Plant Sci. 2017;8:239.

22. Liu D, Dong $X$, Liu Z, Tang J, Zhuang $M$, Zhang $Y$, et al. Fine mapping and candidate gene identification for wax biosynthesis locus, BoWax1 in Brassica oleracea L. var. capitata. Front Plant Sci. 2018;9:309. https://doi.org/10.3389/ fpls.2018.00309.

23. Pu Y, Gao J, Guo Y, Liu T, Zhu L, Xu P, et al. A novel dominant glossy mutation causes suppression of wax biosynthesis pathway and deficiency of cuticular wax in Brassica napus. BMC Plant Biol. 2013;13(1):215. https://doi. org/10.1186/1471-2229-13-215.

24. Zhang X, Liu Z, Wang P, Wang Q, Yang S, Feng H. Fine mapping of BrWax 1, a gene controlling cuticular wax biosynthesis in Chinese cabbage (Brassica rapa L. ssp. pekinensis). Mol Breed. 2013;32(4):867-74. https://doi.org/10.1 007/s11032-013-9914-0.

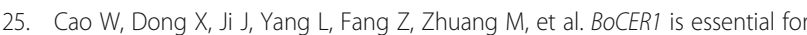
the synthesis of cuticular wax in cabbage (Brassica oleracea L. var. capitata). Sci Hortic. 2020;277:109801.

26. Anstey TH, Moore JF. Inheritance of glossy foliage and cream petals: in green sprouting broccoli. J Hered. 1954;45(1):39-41. https://doi.org/10.1093/ oxfordjournals.jhered.a106433.

27. Farnham MW. Glossy and nonglossy near-isogenic lines USVL115-GL, USVL115-NG, USVL188-GL, and USVL188-NG of broccoli. HortScience. 2010; 45(4):660-2. https://doi.org/10.21273/HORTSCl.45.4.660. 
28. Belser C, Istace B, Denis E, Dubarry M, Baurens FC, Falentin C, et al. Chromosome-scale assemblies of plant genomes using nanopore long reads and optical maps. Nat Plants. 2018;4(11):879-87. https://doi.org/10.103 8/s41477-018-0289-4.

29. Han F, Yuan K, Kong C, Zhang X, Yang L, Zhuang M, et al. Fine mapping and candidate gene identification of the genic male-sterile gene $m s 3$ in cabbage 51S. Theor Appl Genet. 2018;131(12):2651-61. https://doi.org/10.1 007/s00122-018-3180-9.

30. Han F, Yang C, Fang Z, Yang L, Zhuang M, Lv H, et al. Inheritance and InDel markers closely linked to petal color gene (cpc-1) in Brassica oleracea. Mol Breed. 2015;35(8):160. https://doi.org/10.1007/s11032-015-0354-x.

31. Livak KJ, Schmittgen TD. Analysis of relative gene expression data using real-time quantitative PCR and the $2^{-\Delta \Delta C t}$ method. Methods. 2001;25(4):4028. https://doi.org/10.1006/meth.2001.1262.

32. Han F, Cui H, Zhang B, Liu X, Yang L, Zhuang M, et al. Map-based cloning and characterization of BoCCD4, a gene responsible for white/yellow petal color in B. oleracea. BMC Genomics. 2019;20:242.

33. Clough SJ, Bent AF. Floral dip: a simplified method for Agrobacteriummediated transformation of Arabidopsis thaliana. Plant J. 1998;16(6):735-43. https://doi.org/10.1046/j.1365-313x.1998.00343.x.

\section{Publisher's Note}

Springer Nature remains neutral with regard to jurisdictional claims in published maps and institutional affiliations.

- fast, convenient online submission

- thorough peer review by experienced researchers in your field

- rapid publication on acceptance

- support for research data, including large and complex data types

- gold Open Access which fosters wider collaboration and increased citations

- maximum visibility for your research: over $100 \mathrm{M}$ website views per year

At $\mathrm{BMC}$, research is always in progress. 